\title{
El ser se hace de muchas maneras
}

\author{
SAMUEL MANUEL CABANCHIK \\ Departamento de Filosofía e Instituto de Filosofía \\ Facultad de Filosofía y Letras \\ Universidad de Buenos Aires \\ dedalito@hotmail.com
}

\begin{abstract}
Resumen: La tradición pragmatista se ha transformado en una especie de molde o andarivel forzoso para el desarrollo del debate filosófico contemporáneo. Pero, como suele ocurrir en estos casos, la doctrina va perdiendo nitidez en favor de una etiqueta aplicada de modo excesivamente rumboso. La ubicuidad resultante habilita extraños maridajes que dificultan la justa apreciación de mucho de lo que en el pragmatismo sigue siendo una auténtica y profunda renovación de la filosofía.

En el presente trabajo pretendo destacar algunas de las principales características del pragmatismo, sobre todo aquellas que constituyen un giro radical en materia de metafísica respecto de la filosofía moderna. En este sentido, argumentaré que el pluralismo irrealista de Nelson Goodman es una consecuencia natural de algunas tesis pragmatistas acerca de los conceptos mencionados. Para ello partiré de un aspecto del análisis ofrecido por Austin acerca del uso de "real" para desarrollar las posibilidades de una visión radicalmente constructiva de lo real.
\end{abstract}

Palabras clave: realidad, pragmatismo, Nelson Goodman, J.L. Austin, arte, ciencia

Los filósofos gustamos de acuñar nuevas etiquetas capaces de abarcar muchos y grandes asuntos de un modo novedoso, con la esperanza de que por esa vía tengamos la ocasión de renovar el ejercicio de nuestro oficio. A tales tipos de etiquetas pertenecen los varios giros de los que se ha hablado en la segunda mitad del siglo xx: lingüístico, hermenéutico, antropológico y ahora pragmático. La palabra "giro" carga allí con el mayor peso, esto es, el de mentar una reorientación masiva del modo de hacer filosofía. Que ya estemos autorizados a hablar de "giro pragmático" o no puede ser materia de controversia, pero no argumentaré en esa dirección ni trataré de precisar el sentido de esa supuesta reorientación. Mi tema específico es el aporte del pragmatismo a ese hipotético giro. Mi pretensión no es hacer aquí un ensayo de historia crítica de las ideas pragmatistas. Me concentraré en ciertos rasgos que me parecen centrales, en especial en lo que concierne a cuestiones tradicionalmente calificadas de metafísicas, como las relativas a la distinción entre lo real y lo irreal y a si la realidad es una construcción simbólica o se le reconoce alguna dimensión independiente de nuestra invención categorial.

En un trabajo anterior ${ }^{1}$ retomé el análisis de "real" que Austin propuso en Sense and Sensibilia. Allí señalé que de los cuatro rasgos que atribuye a

\footnotetext{
1 "Aspectos semánticos del uso de 'real'”, publicado originalmente en Areté, vol. X, no. 2,
} 
la palabra - a saber, ser una palabra "hambrienta de sustantivo", "de pantalones", "dimensional" y "ajustadora"-, el segundo y el cuarto guardan una relación tensa y estrecha. Austin explica el significado de su expresión "de pantalones" aduciendo que en el uso de "real", quien lleva los pantalones, esto es, el contexto rector o paradigmático, es el negativo. Combinado con el primer rasgo, el modelo resulta ser la expresión tipo "esto no es un $x$ real". Por su parte, su naturaleza ajustadora queda caracterizada a través de la conjunción de dos fórmulas: "esto es como un $x$, pero no es un $x$ real". Austin no es muy claro en su desarrollo de esta característica, pues no afirma con claridad que el ajuste se ejerza a través de la conjunción. Por el contrario, dice que a veces preferimos afirmar la primera parte, y otras, la segunda, y a continuación deja abierta una cuestión en mi opinión importante y sorprendente, al observar que determinar exactamente por qué a veces preferimos decir "es como un $x$ " y otras "no es un $x$ real" constituiría un gran paso en la clarificación del significado de "real".

A fin de apreciar con mayor nitidez el problema que hay aquí, propongo replantear el interrogante de Austin en términos de por qué a veces usamos "real" en su función negativa y otras como palabra ajustadora. En su uso negativo, "real" sirve para establecer un contraste entre un concepto en su uso ejemplar, según el cual el $x$ del caso es la entidad real, y otros ejemplares de $x$ no reales, pero de los que sabemos o podemos saber de qué modo preciso no son $x$ reales. Por el contrario, usamos "como" en su función ajustadora, cuando sólo estamos en condiciones de descartar que la entidad del caso sea un $x$ real, pero no tenemos todavía una etiqueta nueva para clasificarla. Nos limitamos entonces a homologar la entidad no clasificada a una entidad ya etiquetada, generalmente en virtud de ciertas semejanzas.

Estimo que Austin dio con un problema de interés y que algunas ideas atribuibles a la tradición pragmatista permiten apreciar mejor la naturaleza del mismo. El asunto más general que se deja entrever es el de cómo comprender la articulación entre los símbolos en su mutua distribución jerárquica y las entidades a las que se aplican. Una cuestión más específica es la de la explicación y ponderación de los cambios conceptuales y su vínculo con lo que llamamos "la realidad". Por esta vía se reabren viejas preguntas en torno del monismo, el dualismo y el pluralismo y de si hay una realidad permanente y absoluta, que es lo que es con independencia de nuestros sistemas simbólicos, o de si por el contrario son éstos los que rigen, crean y recrean constantemente lo real.

Para avanzar, demorémonos en la complejidad implicada en el uso negativo de "real". Allí tenemos un símbolo que refiere a una clase de cosas, una clasificación de estas cosas en reales y no reales y una clase abierta de

1998, pp. 217-239, y reproducido en U. Pinheriro, M. Ruffino y P. Junqueira Smith (comps.), Ontología, Conhecimento e Linguagem, MAUAD, Río de Janeiro, 2001, pp. 33-49. 
modos de no ser reales esas cosas. Sea la expresión "esto es una estrella". Si digo de una entidad cualquiera que es un ejemplar de estrella, lo que digo es que se trata de una estrella real; pero si digo que no es una estrella real, lo que implico es que es una muestra de estrella de otro género; por ejemplo, de juguete. Ésta es una clase abierta porque pueden aparecer nuevos modos en que algo no sea una estrella real. Hace un tiempo no teníamos expresiones como "un holograma de estrella" o "un modelo computarizado de estrella", por citar algunos ejemplos recientes. Ahora surge la oportunidad para el interrogante de Austin: ¿por qué elijo decir "no es una estrella real, sino una de juguete" y no "es como una estrella, pero no es realmente una estrella"? Una parte de la respuesta es simplemente que hemos decidido extender el uso del símbolo "estrella" a muchas otras entidades que no son estrellas, pero que se presentan con forma de estrella. Naturalmente, los límites de esta extensión son borrosos. El uso del símbolo "estrella" es muy extendido para casos muy variados. Piénsese en las estrellas fijas, en el uso de la expresión "estrella fugaz", en las estrellas de mar, en los cometas llamados también "estrellas de rabo", en dibujos de estrellas de tres, cuatro y cinco puntas, en la Estrella de David, en la expresión "vi estrellas" después de sentir un dolor intenso, en el lucero de la tarde, en el sol, en usos metafóricos como "Maradona es una estrella del fútbol", etc. (dicho sea de paso, obsérvese que, excepto este último ejemplo, todos los demás son usos literales del símbolo, a pesar de no aplicarse a estrellas reales).

Pero esto es sólo una parte de la respuesta, pues ¿por qué ante un cuerpo celeste hasta ahora inobservado puedo decir tanto "es como una estrella, pero no es una estrella" como "no es una estrella real sino una metamorfosis de estrella o algo así"? La respuesta pragmatista, en mi interpretación, desde Peirce a Goodman es: porque así hemos elaborado nuestro sistema simbólico y este sistema en su funcionamiento determina qué es una estrella real, qué una estrella no real y qué no es una estrella. Después de todo, hubo un tiempo en que el Sol no fue una estrella y ya estamos en otro en que sí lo es. Nuestra teoría astronómica podría haber cambiado de modo diferente, o aun podría hacerlo y llevarnos a volver a clasificar al Sol como otro tipo de entidad.

Arribamos así al difícil y conocido terreno de las controversias acerca del realismo. Una bastante citada es la que sostuvieron Goodman y Scheffler y sobre la que también se pronuncia Putnam. Contra la afirmación de Goodman de que nosotros hacemos mundos, que no hay mundo que no hayamos hecho y de que todo en nuestros mundos lo hemos hecho nosotros, Scheffler alega que seguramente no hemos hecho las estrellas, ante lo que Goodman pide un solo rasgo de las estrellas que no hayamos hecho nosotros. Putnam interviene en la discusión argumentando contra Goodman que hay una diferencia fundamental entre, por ejemplo, las estrellas, las constelaciones de estrellas y el uso de un nombre propio para una cons- 
telación en particular. El argumento es que la extensión del nombre de una constelación puede ser fijada por enumeración: si conozco las estrellas de la constelación, tengo la extensión del término. En el otro extremo, el término "estrella" no adquiere su extensión por enumeración. Putnam no está seguro finalmente si para "constelación" vale o no la pretensión de Goodman. En consecuencia, concluye Putnam, es aceptable afirmar que a tal grupo de estrellas nosotros las hemos convertido convencionalmente en una constelación o al menos en esa constelación específica, pero no es cierto que hayamos hecho estrellas a las estrellas. En una palabra, Putnam acepta que por borrosos que sean los límites de la aplicación de un término como "estrella" y por más que contenga desde luego elementos convencionales, es el mundo el que ha hecho estrellas a las estrellas. En todo caso, no sin nuestra ayuda, pues, como él mismo ha dicho alguna vez, "la mente y el mundo construyen conjuntamente la mente y el mundo". ${ }^{2}$

$\mathrm{El}$ argumento de Putnam no prueba lo que él pretende. Del hecho de que el término "estrella" no funcione como un nombre propio o de que no podamos determinar por enumeración su extensión, no se sigue que tenga sentido distinguir en su concepto entre aquello que depende convencionalmente de nosotros y aquello que no. Y el punto de Goodman es ése: tal distinción es imposible. Otros menos extremos, como Clarence Lewis, dirán que hay una dicotomía, pero que es analítica y abstracta, no real. ${ }^{3}$ Para Goodman no hay diferencia esencial entre "constelación" y "estrella", punto en el cual, después de todo, el propio Putnam vacila, aunque en sentido inverso al de Goodman. Pero veamos la cuestión con mayor detenimiento y amplitud.

Lo que está en discusión nos remonta hasta los comienzos mismos de la filosofía. Podemos leer las distintas variantes presocráticas, la teoría de las ideas de Platón y su concepción del receptáculo (khôra) y las teorías aristotélicas del compuesto materia/forma y de las seis causas, como distintos modelos para enfrentar la cuestión de cómo dar cuenta de la articulación última entre lo simbólico —el logos, la razón-y lo real. La filosofía moderna, en particular a partir del giro crítico kantiano, y luego la dialéctica hegeliana son otras tantas alternativas para alcanzar el mismo objetivo. Esquemáticamente, la novedad pragmatista puede interpretarse como un proyecto para resolver ese problema superando tanto los dualismos clásicos y modernos como el monismo hegeliano.

Para comprender y evaluar ese proyecto, a riesgo de abusar de un modo esquemático de exposición, podemos formular la alternativa dualista como

\footnotetext{
${ }^{2}$ Razón, verdad e historia, Tecnos, Madrid, p. 13. Para discusiones acerca del hacer estrellas y el argumento de Putnam, véase P. McCormick, Starmaking, The MIT Press, Cambridge, 1996.

${ }^{3}$ Véase Mind and the World-Order, Dover Publications, Nueva York, 1929, cap. 1.
} 
el reconocimiento de una dicotomía irreductible entre un dato ensimismado y una proyección o una respuesta conceptual frente a ese dato. ${ }^{4}$

Atribuiré al pragmatismo, entonces, el rechazo de esa dicotomía, sean cuales fueren las variantes en las que se deje presentar. Este rechazo es doble: por un lado, toma forma en la negativa a considerar significativa la idea de que pueda pensarse o concebirse algo informe, esto es, un dato puro, no determinado por conceptos o categorías. En otras palabras, para el pragmatismo no hay dato ensimismado, pues todo dato se da originariamente envuelto ya en el movimiento del símbolo — de la semiosis, diríamos con Peirce. En segundo lugar, no habría nada que pueda concebirse bajo la figura de un pensamiento puro, que se capte a sí mismo en su dimensión de acto, sin que entrañe un movimiento de reacción ante el dato ya categorizado y conceptualizado. El concepto más apropiado para pensar el estado de cosas resultante de este doble rechazo es el de situación, como propone Dewey. Entendamos por situación el complejo interactivo entre el sujeto y su entorno. Desde su perspectiva, la realidad misma es en situación. Y toda situación supone la acción de un sujeto orientado hacia fines, lo que desemboca en el privilegio que todos los pragmatistas atribuyen a la acción. A su vez, la realidad de cada cosa dada en el seno de la situación depende de la eficacia que la cosa del caso aporta a la misma, por lo que nada que no posea alguna eficacia contará como real; es decir, para el pragmatismo lo ineficaz no tiene sitio.

He presentado en forma muy comprimida y algo brutal lo que en mi perspectiva constituye un núcleo estratégico del pensamiento pragmatista. Si se acepta este núcleo, no es fácil escapar a las conclusiones más extremas que se encuentran en el irrealismo de Goodman. Me refiero a la idea de que lo real es una creación simbólica. Creación plural, por otra parte, según sean los fines involucrados. Así, la moral, la política, la ciencia, el arte y la filosofía pueden verse como diversas formas en las que creamos y recreamos constantemente la realidad. De semejante constructivismo extremo hay antecedentes incluso en Peirce, quien, sin embargo, dentro de la tradición pragmatista fue el que hizo mayores esfuerzos por permanecer realista. También en Lewis, en quien el pluralismo constructivo es evidente, a pesar de su notable empeño en afirmar un realismo compatible con el relativismo. Precisamente de Lewis podrían citarse muchos pasajes en los

\footnotetext{
${ }^{4}$ Este esquema es más apropiado para la interpretación del pensamiento moderno que del clásico. En particular, no es aplicable sin más a Platón y menos aún a Aristóteles. No puedo justificar ahora esta reserva, pero me excuso de ello señalando que interpreto el pragmatismo como una reacción crítica que se levanta fundamentalmente ante algunos rasgos de la filosofía moderna más que en referencia a la tradición clásica. Tendré que dejar para otra ocasión la tarea de mostrar qué podría leerse en la "originalidad" del pragmatismo, no sin profundas distancias, como la recuperación de muchos elementos de las filosofías de Platón y de Aristóteles. Asimismo, no es mi interés en esta ocasión mostrar en forma directa las diferencias entre el pragmatismo y la filosofía de Hegel.
} 
que el núcleo de ideas al que me he referido aparece prístino. Para muestra van estos botones:

Cualquier experiencia particular es un todo dentro del cual esa parte o aspecto que representa la actividad legislativa o categorial de la mente y aquella que es el contenido dado, independientemente de la interpretación mental, son separables sólo a través del análisis. [...]

El contenido de la experiencia no puede evaluarse o interpretarse a sí mismo. $[\ldots]$

El mundo de experiencia no es dado en la experiencia: es construido por el pensamiento a partir de los datos de la sensación. [...]

Nuestras categorías son guías para la acción. Aquellas actitudes que pasen la prueba de la práctica representarán no sólo la naturaleza de la criatura activa, sino también el carácter general de la experiencia a la que ella se enfrenta. ${ }^{5}$

Para pasar de Lewis a Goodman sólo basta una teoría claramente constructivista de la percepción. Desde luego, ésta no es una diferencia menor, pero ha de reconocerse que la apelación a los datos de la sensación en Lewis es problemática, pues si, como afirma, nuestra experiencia es ya la de un mundo construido por nuestra invención conceptual, regulada a su vez por su eficacia de cara a los fines de nuestra acción, y si la separación del contenido sensorial y los conceptos, las clasificaciones y las categorías es producto de una abstracción que no se proyecta en una polaridad real en la experiencia, no se ve bien qué papel puede desempeñar "lo sensorio puro" allí.

Pero, como dije desde un comienzo, no quiero desarrollar una historia crítica de las ideas pragmatistas. Volvamos entonces a las distinciones y la pregunta de Austin a la luz de lo que presenté esquemáticamente como núcleo estratégico del pragmatismo. En primer lugar, podríamos decir que "real" es una etiqueta o símbolo cuya función es clasificar otros símbolos organizados en un sistema. Así, clasificamos el símbolo "estrella" como un nombre común para referirnos a una clase de entidades reales, y cuando queremos negar que tal símbolo se aplica a algo dado, pero manteniéndolo como miembro del conjunto de las cosas a la que le aplicamos el símbolo "estrella", le adjuntamos un complemento que indica un uso extensivo del símbolo a fin de aplicarlo a muchos otros tipos de cosas asociados al símbolo en cuestión por ciertas semejanzas entre las entidades abarcadas. Se constituye de este modo lo que podríamos llamar una "familia" de símbolos que organizan la experiencia de cierta manera, en función de ciertos fines y en el contexto de ciertas situaciones. Para seguir con el mismo

${ }^{5}$ Ibid. 
ejemplo, digamos que "estrella" es el símbolo que preside la familia asociado a algún ejemplar paradigmático, y en relación con él se organizan los otros miembros de la familia de las estrellas: dibujos, juguetes, etcétera.

En segundo lugar, claro está, este símbolo no se aplica a las entidades organizadas por otras familias de símbolos, a no ser metafóricamente. Por ejemplo, no decimos de un planeta que es una estrella apagada o móvil, o algo por el estilo. Pero nuestro sistema conceptual podría ser diferente de como es si nuestra experiencia, en función de nuestros fines, así nos lo indicara. Por ejemplo, el símbolo "sol" pasó de la familia de los planetas a la de las estrellas. Precisamente respecto del cambio conceptual se vuelve especialmente significativa la característica ajustadora de "como" y su contraste con la función negativa de "real" en los contextos indicados. Frente a un nuevo modo de percibir el Sol en relación con los otros cuerpos celestes, la astronomía copernicana nos llevó a decir en algún momento "no es realmente un planeta"; quizá luego de un tiempo se pudo decir "es como un planeta", para más tarde decir "es una estrella". Otro ejemplo: frente al llamado "oro de los tontos" se habrá dicho "es como el oro, pero no es realmente oro". Otro aún: en algún momento, el flogisto dejó de ser algo real para pasar a ser algo irreal. ¿Qué es lo que a veces nos lleva, pregunta Austin, a contentarnos con negar de un objeto que sea una muestra del ejemplar real y a ponerlo como algún otro miembro de la misma familia, extendiendo entonces el alcance del término original, y otras veces, en cambio, optamos por acentuar que si bien la muestra problemática se parece al ejemplar de referencia, no es realmente una muestra de él? ¿Y cómo, una vez que se empieza a negar de algo que sea realmente una muestra de tal o cual, puede llegarse finalmente a la calificación de "irreal" de ese algo y a la invención de nuevos conceptos e incluso nuevas categorías?

Creo que lo relevante de estas cuestiones está, sobre todo, en el hecho de que en estos casos en que aparece la necesidad de reorganizar nuestro sistema conceptual —el aspecto ajustador de la combinación de "como" y de "no ser real" - lo que resulta problemático es cómo es esto posible si ningún dato de la experiencia se presenta más acá o más allá de las formas que lo determinan como siendo lo que es. ¿Acaso estas situaciones nos obligarán a aceptar que, después de todo, lo informe, el dato puro, irrumpe en nuestra experiencia ya categorizada para presionar por un cambio?

El pragmatista tenderá a responder que para que un dato nuevo cobre alguna significación, debe ser apreciado con nuestros lenguajes y teorías a partir de nuestras necesidades, nuestros intereses y nuestras expectativas, por lo cual no hay tal dato ensimismado. Según su punto de vista, no es que en esas situaciones haya una experiencia de la realidad tal cual es y era desde siempre, contra un lenguaje y una teoría que estaban simplemente erradas. Por el contrario, atribuirá el cambio a una reorientación de nuestra 
"actitud interpretativa", para usar la expresión de Lewis, y agregará que las motivaciones para tal cambio pueden ser muy variadas, y entre ellas se incluirá la nueva experiencia cuya aparición se conformó a través de determinada evolución social y cultural.

La apreciación de las transformaciones de los movimientos artísticos podría prestar un apoyo interesante a este tipo de respuesta. En efecto, nadie diría que para dar cuenta del paso de, digamos, el barroco al clásico, ha debido mediar el encuentro con un dato puro e inesperado o algo así. Es bastante natural que nos expliquemos esos cambios a partir de la libre expresión y creación humanas, tal vez añadiendo que han estado condicionados y orientados por otros cambios más globales en las sociedades. Ahora bien, podría razonar nuestro pragmatista, ¿por qué este tipo de consideración sería sólo aplicable al arte y no a la ciencia o a la filosofía?

El contraste y hasta la oposición entre el arte y la ciencia ha sido muy frecuente en la historia de la filosofía. Es una herencia de Platón y Aristóteles. Baste recordar de este último su Ética a Nicómaco, donde se afirma que los caminos del alma para alcanzar la verdad son, entre otros, la ciencia y el arte, pero mientras la primera es teoría y contemplación puras de las verdades necesarias y eternas, el segundo, que abarca la producción y la acción, nos remite a lo contingente y mudable. El arte productivo en particular, diferenciado por Aristóteles radicalmente de la acción, aunque racional, rige las cosas que no existen por sí y que como tales no son ni necesarias ni naturales.

Respecto de este cuadro de situación, el pragmatismo constituye un cambio muy profundo, con base en el cual se destacan las similitudes y continuidades entre ciencia, arte y acción. En efecto, los criterios de formación y corrección de los conceptos son patrones de acciones reales o posibles y la ciencia no es menos "arte productivo" que lo que éste a su vez puede ser vehículo de conocimiento y de verdad. Para ilustrar en forma directa este cambio, recuérdese que Dewey observa que si la filosofía griega está equivocada sobre esta materia, entonces sería cierto que la ciencia es arte, que el arte es práctica y que la única distinción digna de ser establecida no es entre práctica y teoría, sino entre aquellos modos de práctica que no son inteligentes ni gozables inherente e inmediatamente, y aquellos que están llenos de significados gozados. Y agrega en tono profético: "De este modo desaparecerían las separaciones que perturban el pensamiento actual: la división de todo en naturaleza y experiencia, de la experiencia en práctica $y$ teoría, arte $y$ ciencia, del arte en útil $y$ bello, servil $y$ libre." ${ }^{\prime 6}$

El punto de vista pragmatista representado por Dewey, y más recientemente por Goodman, lleva inevitablemente al rechazo del empirismo realista de inspiración lockeana. Junto a las dicotomías señaladas por Dewey

\footnotetext{
${ }^{6}$ En Experience and Nature, reproducido en Pedagogía y filosofía, Francisco Beltrán, Madrid, 1950, pp. 492 ss.
} 
en el pasaje precedente, desaparecen las de apariencia/realidad, propiedades en sí/propiedades proyectadas, etc. De acuerdo con esta perspectiva, la ciencia no puede seguir siendo concebida entonces como el instrumento para conocer una realidad trascendente ensimismada. Su naturaleza se asemeja a la del arte en que ambos son instrumentos de renovaciones conceptuales y categoriales de la experiencia a partir de las cuales se alcanza la creación y recreación de lo real.

Desde luego, esto no nos lleva a una indiferenciación masiva del arte y la ciencia; su diferenciación pasará ahora por sus distintas funciones y por algunas características de sus sistemas simbólicos. Mientras que en las ciencias importan la verdad, la denotación, la explicación y la predicción, en el arte se enseñorean la metáfora, la ejemplificación y la expresión. Pero, a los efectos del tema que nos ocupa, es más importante para nosotros aquello en que arte y ciencia se aproximan que aquello en lo que difieren. En este sentido, la novedad conceptual implícita en el uso ajustador de "real" no nos llevaría necesariamente al reconocimiento de una instancia en la que ingresaría pasivamente en la experiencia un contacto con una realidad trascendente. El cambio conceptual, sea en el nivel del lenguaje natural o del lenguaje de las teorías científicas, podría ser interpretado como la introducción activa de una reorganización del campo de la experiencia, gobernada por hábitos y experiencias previas, y dirigido por la meta de arribar a representaciones cada vez más ajustadas e integradas, de modo de que se adquiera una mayor capacidad de unificación de la experiencia.

Ahora bien, aun si se aceptara la interpretación del conocimiento en términos de creación, no por ello se habría establecido la corrección de que "no hay nada que no hayamos hecho nosotros". En primer lugar, no debe olvidarse que ninguna de las posiciones posibles en esta discusión admitirá la creación ex nihilo. Quiere decir que siempre partimos de una situación dada en la que el dato aparece al menos como ya hecho. Entonces, el "nosotros" de la frase "no hay nada que no hayamos hecho nosotros" debe interpretarse como "los seres humanos", "los sistemas simbólicos o esquemas conceptuales", "una mente en general", o similares; es decir, en un sentido muy estrecho de "nosotros" sí hay muchas cosas que no hemos hecho: todo aquello que es un dato ya conformado por la acción de otros y de sistemas simbólicos ya dados. Sólo en un sentido muy amplio, general e indeterminado del "nosotros" podría por ahora admitirse la corrección del pensamiento que nos ocupa.

Pero los problemas no terminan ahí. La premisa principal del argumento de Goodman y otros pragmatistas o neopragmatistas es que no hay modo de distinguir en dato alguno aquello que depende de nuestras actividades, nuestros intereses y nuestras convenciones, de aquello que cuente como un contenido o sustancia cuya realidad no sea relativa a nosotros en ningún sentido relevante. Pero si esta distinción es un sinsentido, también lo será 
su negación. Para plantear el problema de un modo significativo, conviene partir de circunstancias en las que tengamos descripciones alternativas que remitan, por una parte, a distintas convenciones, y, por otra, a "las mismas cosas" o "los mismos hechos". Podemos recordar el ejemplo de Putnam en el que se confrontan dos descripciones de una situación dada: una en términos de individuos identificados por los medios comunes de percepción y de lenguaje y otra en términos mereológicos a partir de los conceptos de parte y todo. Si tengo tres objetos sobre la mesa de acuerdo con la primera descripción, tendré siete u ocho — según se incluya un "objeto nulo" o node acuerdo con la segunda. A continuación, el pragmatista deberá negar que haya un modo en que el mundo es en sí, mientras que el empirista realista insistirá en que, tengamos o no una descripción neutral de él, las descripciones alternativas lo son de un mismo y único mundo. La consecuencia es que para el pragmatismo las categorías de individuo, objeto, identidad y realidad pueden adquirir múltiples significaciones según sean nuestros criterios y nuestras convenciones.

Otro ejemplo ilustrativo para apreciar el problema es el conocido como "el barco de Teseo". Recordémoslo brevemente. Supongamos un barco bautizado "Teseo" al que se le cambian todas sus partes por otras de otro material pero conservando sus propiedades funcionales. Al cabo del cambio completo nos queda un barco que, según sean nuestros criterios de identidad, es el mismo que el original o es otro. Llamémoslo "Teseo 1" e imaginemos que se han guardado todos las partes originales con las que luego se construye un barco idéntico al original. Éste será nuestro "Teseo 2". Tenemos como resultado dos barcos y cabe la cuestión de cuál de los dos es "Teseo". Está claro que la respuesta depende enteramente de nuestras convenciones, por lo cual la identidad de un individuo se vuelve tan relativa como la clase de cosa que es según nuestros conceptos.

Al volver ahora a la cuestión de si hemos hecho o no a las estrellas, una tesis como la goodmaniana no sólo dice que hemos hecho esa clase de cosas, sino que debe afirmar que la existencia misma de cada estrella en su individualidad es conceptualmente dependiente de nuestras convenciones, en este caso, de nuestros criterios de identidad. Desde luego, reconoce Goodman, no hacemos estrellas como el obrero fabrica ladrillos, es decir, no las hemos hecho materialmente, pero sí las hemos hecho conceptualmente y en un sentido literal, no metafórico. En cuanto a la materia misma, está claro que su identificación en cada caso como una muestra de tal o cual tipo vuelve a ser conceptualmente dependiente. Tampoco la materia como tal la creamos materialmente, pero sí conceptualmente. En consecuencia, la distinción entre forma y contenido o cualquier otra similar, si bien necesaria, será relativa y variable.

Hasta aquí he intentado mostrar el sentido de la interpretación pragmatista de la distinción entre lo real y lo irreal y su análisis del funcionamiento 
de nuestros sistemas simbólicos en su alcance metafísico. Si mi lectura es correcta, el pluralismo irrealista de un Goodman es un extremo coherente al que arriban las posiciones pragmatistas. Si así ocurre es porque, una vez eliminadas las bases para las dicotomías centrales de la filosofía moderna, especialmente en sus versiones empiristas, no queda dique en pie que impida concluir que lo que determinamos como real es dependiente de nuestros conceptos y categorías. Más aún, deberá admitirse que no hay argumento concluyente para evitar afirmar que lo real es una construcción nuestra y que el conocimiento es creación.

Tal es el estado de cosas al que nos lleva la crítica pragmatista de las dicotomías heredadas de la tradición moderna. Aunque creo correcta la orientación pragmatista y pienso que en efecto logra superar muchas aporías de la filosofía moderna, me parece que el problema al que aludí a partir de la distinción y la pregunta planteadas por Austin aún requiere un tratamiento más amplio. Hasta ahora sólo tenemos razones para evitar una interpretación realista en los términos dicotómicos antes referidos. Pero una explicación que no dé cuenta del modo en que se articulan el dato novedoso que rebasa nuestras clasificaciones y éstas mismas en su funcionamiento inmanente y regimentado, seguirá siendo insuficiente.

Esta insuficiencia se aprecia en el planteamiento del mismo Austin. Como indiqué al comienzo, hay tensión no resuelta entre la función "de pantalones" y la función de ajuste atribuidos al predicado "ser real". Es hora de hacer precisiones al respecto. La tensión está dada por lo siguiente: según la función "de pantalones", usamos "no es un $x$ real" cuando ya está establecida la clasificación entera que distribuye los roles dentro de una "familia" determinada, incluido el papel principal de ser el paradigma de lo real para esa familia, y usamos la misma expresión "no es un $x$ real" cuando necesitamos ajustar nuestros símbolos en el curso de una situación que lo requiere. En verdad ejercemos constantemente ambas funciones: una para conservar y afianzar lo que ya tenemos, la otra para renovar y crear lo que no tenemos.

La pregunta que se hace Austin en relación con la función de ajuste, acerca de por qué a veces decimos "no es un $x$ real" y a veces "es como un $x$ ", se relaciona con la oscilación que se da en nuestras prácticas entre tendencias conservadoras y renovadoras. Austin muestra su tendencia conservadora al hacer descansar la función ajustadora de "real" en la de "como". Pero en verdad ésa es sólo una de las caras del ajuste. La otra cara muestra lo que quizá sea el aspecto principal, que es el renovador. A través de la fórmula negativa "no es un $x$ real", podemos querer conservar el status quo, así como establecer una nueva taxonomía. Una vez establecida, según sea el mayor o menor grado de profundidad del ajuste, puede resultar una compleja y completa redistribución de familias enteras de conceptos. 
Entonces la función "de pantalones" reaparecerá una vez más, ahora para realizar las distinciones en el interior del nuevo status quo.

Esta polarización de tendencias se refleja en las estructuras simbólicas - o es constituida por ellas-, pues para que haya simbolización y cognición es necesario que haya símbolos que funcionen categóricamente para fijar el contenido, mientras se deja libertad a otros que pluralizan las posibles versiones de ese contenido. Si bien la distinción entre convención variable y contenido fijo es relativa o, si se prefiere, ella misma se traza convencionalmente, también es cierto que cualquier "versión de mundo" debe hacer factible la distinción entre ambos elementos. Respecto de muchas versiones será cierto que versan sobre los mismos hechos o las mismas cosas. Claro que el contenido común también es conceptualmente dependiente de esas mismas versiones y de otras. Lo que importa es reconocer, sin temor a reinstalar las viejas dicotomías, pues no es el caso, que para cada versión habrá siempre una "materia" que ella no hace y con la que hace el mundo del que es versión. Esa materia, a su vez, habrá sido hecha por otras versiones y no es posible — ni tiene sentido pretenderlo- remontarse a una situación de origen. ${ }^{7}$

Conservación de lo que existe como existe y transformación revolucionaria son fuerzas que trabajan constantemente nuestras teorías y nuestras prácticas. Virtualmente, todos somos en mayor o menor grado conservadores y revolucionarios. Pero, independientemente de las batallas políticas involucradas - hablo de política conceptual tanto como de la otra-, son los sistemas simbólicos mismos los que funcionan así. Seguramente no hay una explicación general de este rasgo, sino que en cada situación habrá una "lógica" que lleva de un estado apacible de nuestros conceptos y categorías a un cambio más o menos profundo para estabilizarse de nuevo. Es oportuno recordar aquí aquella idea de Peirce de que hay un movimiento continuo, un proceso de inferencia inconsciente que irrumpe en abducciones y cristaliza en juicios de percepción, los que a su vez fungen como base dentro del proceso semiótico racionalmente controlado. Como hipótesis metafísica, entonces, puede aventurarse esta idea de procesos inconscientes que constituyen un motor para las transformaciones, y cuya eficacia y valor dependerán de las capacidades constructivas de los sistemas simbólicos que sepamos construir.

Ahora el título de este trabajo puede ser repetido como conclusión. “¿Qué es lo que hay?", pregunta la ontología. La respuesta es que hay lo que hacemos existir de múltiples maneras con una variedad de instrumentos, ellos mismos en crecimiento y transformación permanentes. Esto no nos vuelve émulos de Dios, pues nuestro negocio no es crear desde la nada y,

${ }^{7}$ Un desarrollo más amplio de este problema puede verse en N. Goodman, Way of Worldmaking, Hackett, Indianápolis, 1978, y N. Goodman y C. Elgin, Reconceptions in Philosophy and Other Arts and Sciences, Hackett, Indianápolis, 1988. 
en la misma medida en que creamos y recreamos mundos, somos por ellos mismos transformados constantemente. Es el sentido que puede dársele a aquella frase de Putnam antes recordada: "la mente y el mundo construyen conjuntamente la mente y el mundo". En definitiva, el ser se hace de muchas maneras en nosotros, con y contra nosotros. Y la filosofía puede encontrar un sentido en su potencial para participar en esta tarea. Estimo que el giro pragmático constituye, en nuestra época, una de las principales herramientas para la orientación del trabajo filosófico dirigido en favor de esta potencia.

Recibido el 14 de enero de 2002; aceptado el 28 de mayo de 2002 